\title{
Gender specific decline in perioperative allergic reactions after withdrawal of pholcodine
}

\author{
Lars Malvik$^{1}$, Gerrit De Pater ${ }^{1}$, Geir Olav Dahle ${ }^{1}$, and Anne Berit Guttormsen ${ }^{1}$ \\ ${ }^{1}$ Haukeland University Hospital
}

July 31,2020

\begin{abstract}
Background: There is a known female predominance in immunological conditions. Gender differences have also been demonstrated in perioperative hypersensitivity. The aim of this study was to investigate gender differences in the incidence of perioperative allergic reactions in Norway from 1997 to 2017. Methods: Spontaneous reports on allergic reactions during anesthesia were collected, and information about demographic, clinical and laboratory findings were recorded. Results: Women account for $66 \%$ of reported cases, and gender difference was most pronounced in fertile women (ages 14 to 51 ), where $74 \%$ of reports were from women. After withdrawal of the only pholcodine containing cough syrup on the market in 2007, the female predominance fell from 72 to $60 \%$ in all age groups. We found no differences in severity of the reaction or in laboratory findings between men and women. Conclusions: Women are at increased risk of suffering perioperative hypersensitivity reactions. The gender difference was less pronounced after the withdrawal of pholcodine.
\end{abstract}

I: TITLE

\section{Gender specific decline in perioperative allergic reactions after withdrawal of pholcodine}

\section{II: SHORT RUNNING TITLE}

Gender difference in perioperative hypersensitivity

III: AUTHORS

Lars Berg Malvik (1)

Gerrit Hendrik De Pater (1)

Geir Olav Dahle (1)

Anne Berit Guttormsen $(1,2)$

IV: AFFILIATIONS

1. Haukeland University Hospital, Bergen, Norway

2. Department of Clinical Medicine, University of Bergen

$\mathrm{V}$ : ACKNOWLEDGMENTS

Erik Florvaag, Professor emeritus, for constructive feedback in the work on this manuscript.

VI: ABSTRACT 
Background: There is a known female predominance in immunological conditions. Gender differences have also been demonstrated in perioperative hypersensitivity. The aim of this study was to investigate gender differences in the incidence of perioperative allergic reactions in Norway from 1997 to 2017.

Methods: Spontaneous reports on allergic reactions during anesthesia were collected, and information about demographic, clinical and laboratory findings were recorded.

Results: Women account for $66 \%$ of reported cases, and gender difference was most pronounced in fertile women (ages 14 to 51 ), where $74 \%$ of reports were from women. After withdrawal of the only pholcodine containing cough syrup on the market in 2007, the female predominance fell from 72 to $60 \%$ in all age groups. We found no differences in severity of the reaction or in laboratory findings between men and women.

Conclusions: Women are at increased risk of suffering perioperative hypersensitivity reactions. The gender difference was less pronounced after the withdrawal of pholcodine.

Keywords: Anesthesia, Drug Hypersensitivity, Pholcodine, Sex Factors

VII: MAIN TEXT (WORD COUNT: 2346)

\section{Introduction:}

There is a known gender difference in immunological diseases ${ }^{1}$. Allergy and anaphylaxis are more frequent in women, with varying reports on the difference in the severity of reactions ${ }^{2,3}$. Women are overrepresented both during their fertile years, and to a lesser degree before menarche and after menopause. This suggests both genetic and hormonal mechanisms ${ }^{4}$. The hormonal contribution to immunological diseases is demonstrated by variations in symptoms during pregnancy, as well as in different phases of the menstrual cycle. ${ }^{5,6}$

For allergic reactions to appear, several conditions need to be met. First, an antigen needs to be introduced before it is picked up by an antigen-presenting cell. This stimulates T-cell differentiation and proliferation, promoting B-cell activation. B-cells produce IgE antibodies specific to the original antigen, the antigenantibody complex triggers receptors on the surface of mast cells, leading to degranulation and release of mediators, most importantly histamine. Mediators cause allergic symptoms from skin, lungs and the cardiovascular system. All these mechanisms are influenced by the levels of sex hormones, shown in vitro and in animal models ${ }^{7-10}$.

Perioperative allergy and anaphylaxis are rare events, and therefore challenging to study. In the recent NAP6 studies, the incidence of a severe reaction is estimated at 1 in 10000 , with a suspected higher true incidence ${ }^{11,12}$. In the NAP6 sub studies there is no focus on gender differences, while some of the works on the French anaphylaxis registry by Mertes et al, demonstrate a female predominance ${ }^{13,14}$. Mertes points to the hypothesis of possible cross-sensitization with quaternary ammonium ion-containing compounds put forward by Florvaag et al $^{15}$, and the use of such compounds in cosmetics, as a possible explanation of this gender difference. The Norwegian Network for Anaphylaxis under Anesthesia (NARA) has published several studies describing the panorama of hypersensitivity reactions in Norwegian operating rooms, both before and after withdrawal of the only pholcodine containing cough syrup from the Norwegian market in $2007^{16,17}$ . The effect of this withdrawal on the level of sensitization of the population was evident, with a significant decline in the number of reports and IgE sensitization from 2007 to $2009^{16}$.

NARA gives us the opportunity to further investigate the influence of gender on perioperative hypersensitivity.

\section{Methods:}

The present study is a retrospective registry study, covering the period from 1997 to 2017, based on standardized reports to NARA, a spontaneous reporting system of suspected perioperative hypersensitivity. 


\section{The NARA database}

NARA has collected standardized data on suspected anaphylactic reactions during anesthesia since 1997. The physician that experienced the reaction reported it and sent blood samples to the Laboratory of medical biochemistry and pharmacology, Haukeland University Hospital. All available information on the reaction was included in the NARA database. Preferably three serum samples taken within 2 and 24 hours after the reaction, and if available, samples taken before the reaction were provided. Serum levels of tryptase and IgE antibodies against pholcodine (PHO), morphine (MOR), suxamethonium (SUX), latex (LAT) and chlorhexidine (HEX) were routinely analyzed using ImmunoCap system (Phadia/Thermo Fisher Scientific, Uppsala, Sweden). All data were stored in FileMaker pro (FileMaker Inc., Santa Clara, CA, USA). Reported cases not related to anesthesia, reports not containing the required information or reactions occurring $>24$ $\mathrm{h}$ after induction of anesthesia were excluded. The patients gave written informed consent. For those who did not reply the ethical committee allowed passive consent. From 1379 consecutive reports we registered year of reaction, age, gender, use of muscle relaxant, acute level of serum tryptase, pre- and post-reaction serum tryptase, total $\mathrm{IgE}$, specific $\mathrm{IgE}$ antibodies, clinical severity of reaction on scale of 1 to 5 according to a modified Ring \& Messmer scale ${ }^{18}$ and whether patients had been allergologically investigated for possible culprit substances or not. The lowest measurement between pre- and post-reaction serum tryptase was considered the base level. A factorial increase of 1.2 plus two units from baseline serum tryptase was considered significant ${ }^{19}$. Severity of the reaction was for some analyses converted in to 'Mild' (1-2) and 'Severe' (3-5). Age group splits were constructed according to the Norwegian national average age of menarche at 13 years ${ }^{20}$ and menopause at 51 years $^{21}$. Specific IgE [?]0.35 kUA/l was considered significant for sensitization. Because many patients had significant increase in more than one IgE antibody, we constructed a variable of the sum of all the IgE antibodies registered (PHO, SUX, MOR, LAT and HEX), as the contribution of each specific subtype to the reaction would be difficult to determine. Normalization of this number and of IgE antibodies with other specificities for total IgE gives us a "specific activity" (also known

as the "allergen-specific IgE to total IgE ratio"), which has been hypothesized to be a better predictor of effector cell activation than IgE antibodies alone ${ }^{22}$.

\section{Statistics}

All data management and analysis was done with ' $\mathrm{R}$ ' ${ }^{23}$ in 'RSTUDIO' ${ }^{24}$. Packages used includes 'dplyr'25 and 'ggplot2' ${ }^{26}$. Significance level was $P<.05$. We explored group differences using Pearson's Chi-squared test for categorical variables. After graphical screening of continuous variables, some were tested for normal distribution using Shapiro-Wilk test. Groups were then compared using Welch two-sample t-test or WilcoxonMann-Whitney test on ranks, whenever appropriate. Quantile function for the binomial distribution was used to compute confidence intervals for non-normal variables. There was no imputation of missing values.

\section{Ethics}

The study was approved by the Regional Committee for Medical Research Ethics in Western Norway $(2009 / 2124)$

\section{Results}

\section{Descriptive statistics}

A total of 1379 reports were subjected to analysis. Age range was 0 to 89 years (median 42 years). Muscle relaxants were used in 1023 patients $(74.2 \%)$. Most reports (91.7\%) included a clinical severity score from 1 to 5 (Table 1 ).

\section{[TABLE 1 NEAR HERE]}

There was a female predominance in all age groups, the effect was most pronounced in fertile women (14-51 years) (Figure 1). In subjects younger than 14 years or older than 52 years, the male to female ratio was 
$44.0 \%$ to $56.0 \%(P<.01)$, respectively. There was no significant difference in reaction severity between men and women, men being reported with $51.2 \%$ 'Mild' reaction and $40.8 \%$ 'Severe' (remainder missing), whilst in women $49.2 \%$ had a 'Mild' and $42.4 \%$ had a 'Severe' reaction $(P=0.52)$. Information on whether patients have been allergologically investigated is available for $42.7 \%$ of women, and $38.4 \%$ of men. In cases where investigation status is known, the proportion of women $(81.9 \%)$ that have been investigated is higher than in men $(67.2 \%)(P<.01)$.

\section{[FIGURE 1 NEAR HERE]}

\section{[TABLE 2 NEAR HERE]}

There was no difference in the increase of serum tryptase normalized to the specific activity of IgE all age groups considered $(P=0.43)$. There was no gender difference in the increase of serum tryptase for the different grades of severity, except for grade 1 reactions. Most patients with less severe reactions did not have a significant increase in serum tryptase (86\%) (Table 3).

\section{[TABLE 3 NEAR HERE]}

\section{Temporal trends}

From 1997 to 2007, there was a mean of 76 reports per year. After 2007 the mean number of reports fell to 61 . There was a period of stable reporting after an initial yearly increase in number of reports following the establishment of the registry. From 2001 to 2007, there was a mean of 87 reports per year. There is a new stability in number of reports from 2009, with a mean of 65 annual reports until 2017. The gender difference was significant in both periods of stable reporting. From 2001 to 2007 there was a $72 \%$ female predominance $(P<.01)$ and from 2009 to $201760 \%$ were women $(P<.01)$ (Figure 2$)$.

\section{[FIGURE 2 NEAR HERE]}

Trends in use of NMBA and elevated IgE antibody levels to SUX and HEX are shown in Table 4.

\section{[TABLE 4 NEAR HERE]}

\section{Discussion}

Complex physiological and immunological processes are needed to produce an allergic reaction. Many of these are under the influence of sex hormones, where estrogen acts both anti- and pro-inflammatory, while testosterone and progesterone are anti-inflammatory ${ }^{6-10,27-29}$. Finding a female predominance in perioperative hypersensitivity is therefore not surprising. The degree to which women are overrepresented prior to the withdrawal of pholcodine is more pronounced than in similar reports ${ }^{2,13}$.

Following the withdrawal of pholcodine from the market in Norway in 2007 there was an observed decline in the incidence of perioperative allergic reactions, from about 90 reports a year to about 60 . The novelty of this study is that most of this reduction seems to have taken place in the female proportion of patients. It might be that exposure to pholcodine stimulates different immunological responses in men and women.

Mertes and collaborators hypothesized that gender differences in perioperative hypersensitivity has a link to pholcodine consumption ${ }^{30}$, but little is known about the underlying mechanisms. A difference in patterns of consumption (between men and women) could explain our findings. From sales numbers Florvaag estimated that $40 \%$ of the Norwegian population has been exposed to pholcodine ${ }^{15}$. It was sold over the counter until 2007, but patterns of use are difficult to assess.

If not explained by behavioral mechanisms there may be a gender difference in the way immunoglobulin producing cell lines react to antigens, and that this difference is more pronounced in fertile than in nonfertile women. This could be a byproduct of some unrelated immune process, or maybe a result of an active immunocontraceptive processes in female reproduction. Pholcodine, morphine and suxamethonium, as well as other NMBAs, are chemically associated by their quaternary ammonium-ion (QAI). The QAIs of 
suxamethonium are the active sites of the molecule. These bind to the acetylcholine (ACh) receptors of the neuromuscular plate, and inhibit the actions of endogenous ACh. This neurotransmitter is thought to be present mostly in vertebrates and insects, but has also been found in varying amounts in plants, fungi and bacteria $^{31}$. There could thus be a theoretical basis for pholcodine sensitization being possibly beneficial in the defense against infections that women are at special risk of acquiring during their fertile years.

We did not find any gender difference in total IgE. There was no significant difference in absolute or relative serum tryptase levels, but conclusions based on this finding are problematic as we do not know exactly at what time blood was drawn relative to the debut of the reaction, and the half-life of serum tryptase is short. There was no difference in the clinical severity of the reaction between men and women; this is in line with the similar biochemical findings.

\section{Limitations}

The fact that our results contradict the hormonal effects on immunology put forward by several other studies is problematic. These studies often investigate the influence of single hormones in models with limited influence by other hormones, which may offer one explanation. Probably it is the balance of sex hormones that dictates the end result, not only individual variations. We did not investigate serum level of hormones in our patients, and we have no information on their use of contraceptives or their pregnancy status. Bodyweight could also be of influence, since adipose tissue is hormonally active. A sudden change in contraceptive use in Norway in 2007, when the dominating cyclic hormonal contraception was largely replaced by monophasic products may also be a confounding factor ${ }^{32}$. On the other hand, as demonstrated

by numbers from the Global Burden of Disease database, there was an increase in the female incidence of other immunological conditions such as asthma and urticaria in Norway during the same time period ${ }^{33}$.

In our dataset, the exact time from the allergic reaction to sampling of blood is unknown. Having a relatively short half-life, time of sampling will influence the concentration of serum tryptase. If there were systematic differences between men and women, this would influence our findings.

There has been some variation in the number of reports to NARA during the 20 years of registration. For some of the analyses on temporal trends we assume that reporting is stable from 2001. If this assumption does not hold true, the effect on the conclusions are important.

It is a weakness that the number of measured $\operatorname{IgE}$ antibodies is limited to five substances. Having the highest number of complete reports, IgE SUX and IgE HEX were chosen to represent QAI and non-QAI compounds, respectively, in the exploration of temporal differences. It seems that the differences are much more pronounced for the QAI group. Sensitization to chlorhexidine and latex is rare in Norway; the lack of significant decrease may be due to rarity, not because a difference does not exist. Data on whether both male and female patient have been allergologically evaluated post reaction, $61.6 \%$ and $57.3 \%$ respectively, are incomplete. Consequently the culprit agent remains unidentified in about half of our patients, and the clinical importance of the $\mathrm{IgE}$ antibody measurements remains unclear.

\section{Conclusion}

Women are overrepresented in the database of the Norwegian Network for Anaphylaxis under Anesthesia, accounting for two thirds of reported cases. After the withdrawal of pholcodine from the Norwegian market, the gender difference has become less pronounced, as there was an isolated female reduction in number of perioperative allergic reactions after 2007. This reduction is accompanied by a decrease in the number of quaternary ammonium-ion-sensitized females, while the men have a more modest reduction in sensitization. These effects are only present in fertile women. Although being rare events, perioperative allergic reactions can have severe consequences. Still ongoing sales of pholcodine containing drugs in many countries continues to represent a threat to patients under perioperative care, especially so to the female population.

VIII: REFERENCES 
1. Klein SL, Flanagan KL. Sex differences in immune responses.Nature reviews. Immunology. 2016;16(10):626-638.

2. Francuzik W, Nassiri M, Babina M, Worm M. Impact of sex on anaphylaxis severity-data from the Anaphylaxis Registry. Journal of Allergy and Clinical Immunology. 2015;136(5):1425-1426.

3. Hox V, Metcalfe DD, Olivera A. Reply. Journal of Allergy and Clinical Immunology. 2015;136(5):1426.

4. Salvati L, Vitiello G, Parronchi P. Gender differences in anaphylaxis. Current opinion in allergy and clinical immunology.2019;19(5):417-424.

5. Skoczynski S, Semik-Orzech A, Szanecki W, et al. Perimenstrual asthma as a gynecological and pulmonological clinical problem. Advances in clinical and experimental medicine : official organ Wroclaw Medical University. 2014;23(4):665-668.

6. Shah NM, Imami N, Johnson MR. Progesterone Modulation of Pregnancy-Related Immune Responses. Frontiers in immunology.2018;9:1293.

7. Bonds RS, Midoro-Horiuti T. Estrogen effects in allergy and asthma.Current opinion in allergy and clinical immunology.2013;13(1):92-99.

8. Hox V, Desai A, Bandara G, Gilfillan AM, Metcalfe DD, Olivera A. Estrogen increases the severity of anaphylaxis in female mice through enhanced endothelial nitric oxide synthase expression and nitric oxide production. The Journal of allergy and clinical immunology.2015;135(3):729-736.e725.

9. Intan-Shameha AR, Abu Bakar MZ, Noordin MM, Haron AW, Azmi TI. The effects of oestrogen and progesterone on lymphocyte and plasma cell population in the oviduct and uterine mucosae during follicular and luteal phases in ewes. Pertanika Journal of Tropical Agricultural Science. 2011;34:181-187.

10. Laffont S, Seillet C, Guery JC. Estrogen Receptor-Dependent Regulation of Dendritic Cell Development and Function. Frontiers in immunology. 2017;8:108.

11. Mertes PM, Ebo DG, Garcez T, et al. Comparative epidemiology of suspected perioperative hypersensitivity reactions. British journal of anaesthesia. 2019;123(1):e16-e28.

12. Harper N, Cook T, Garcez T, et al. Anaesthesia, surgery, and life-threatening allergic reactions: epidemiology and clinical features of perioperative anaphylaxis in the 6th National Audit Project (NAP6).British journal of anaesthesia. 2018;121(1):159-171.

13. Mertes PM, Alla F, Tréchot P, Auroy Y, Jougla E. Anaphylaxis during anesthesia in France: An 8-year national survey. Journal of Allergy and Clinical Immunology. 2011;128(2):366-373.

14. Mertes PM, Volcheck GW, Garvey LH, et al. Epidemiology of perioperative anaphylaxis. Presse medicale (Paris, France : 1983). 2016;45(9):758-767.

15. Florvaag E, Johansson SG. The pholcodine story. Immunology and allergy clinics of North America. 2009;29(3):419-427.

16. de Pater GH, Florvaag E, Johansson SG, Irgens A, Petersen MN, Guttormsen AB. Six years without pholcodine; Norwegians are significantly less IgE-sensitized and clinically more tolerant to neuromuscular blocking agents. Allergy. 2017;72(5):813-819.

17. Florvaag E, Johansson SG, Irgens A, de Pater GH. IgE-sensitization to the cough suppressant pholcodine and the effects of its withdrawal from the Norwegian market. Allergy. 2011;66(7):955-960.

18. Kroigaard M, Garvey LH, Gillberg L, et al. Scandinavian Clinical Practice Guidelines on the diagnosis, management and follow-up of anaphylaxis during anaesthesia. Acta anaesthesiologica Scandinavica. 2007;51(6):655-670. 
19. Cook TM, Harper NJN, Farmer L, et al. Anaesthesia, surgery, and life-threatening allergic reactions: protocol and methods of the 6th National Audit Project (NAP6) of the Royal College of Anaesthetists. British journal of anaesthesia. 2018;121(1):124-133.

20. Menarche. (2019, July 11). Norwegian Medical Encyclopedia . https://sml.snl.no/menarke.

21. Menopause. (2019, July 11). Norwegian Medical Encyclopedia . https://sml.snl.no/menopause.

22. Hamilton RG, MacGlashan DW, Jr., Saini SS. IgE antibody-specific activity in human allergic disease. Immunologic research.2010;47(1-3):273-284.

23. R: A language and environment for statistical computing. [computer program]. R Foundation for Statistical Computing, Vienna, Austria; 2007.

24. RStudio: Integrated Development for $R$ [computer program]. RStudio, Inc., Boston, MA; 2015.

25. dplyr: A Grammar of Data Manipulation [computer program]. Version 0.8.3. https://CRAN.Rproject.org $/$ package $=$ dplyr2019.

26. ggplot2: Elegant Graphics for Data Analysis [computer program]. Springer-Verlag New York; 2016.

27. Khan D, Ansar Ahmed S. The Immune System Is a Natural Target for Estrogen Action: Opposing Effects of Estrogen in Two Prototypical Autoimmune Diseases. Frontiers in immunology. 2015;6:635.

28. Shah S. Hormonal link to autoimmune allergies. ISRN allergy.2012;2012:910437.

29. Ziegler SM, Feldmann CN, Hagen SH, et al. Innate immune responses to toll-like receptor stimulation are altered during the course of pregnancy. Journal of reproductive immunology. 2018;128:30-37.

30. Mertes PM, Tajima K, Regnier-Kimmoun MA, et al. Perioperative anaphylaxis. The Medical clinics of North America.2010;94(4):761-789, xi.

31. Horiuchi Y, Kimura R, Kato N, et al. Evolutional study on acetylcholine expression. Life sciences. 2003;72(15):1745-1756.

32. Sakshaug S (red) OK, Berg C, Blix HS, Dansie LS, Litleskare I, Granum T. Legemiddelforbruket i Norge 2014-2018 [Drug Consumption in Norway 2014-2018]. Oslo: Folkehelseinstituttet2019.

33. Global Burden of Disease: Incidence of Asthma and Urticaria for men and women aged $\quad 15-49 \quad$ years. 1997-2017; http://ghdx.healthdata.org/gbd-results-tool?params=gbd-api-2017permalink/b7a801971276e5334061180d00af9b5c.

\section{IX: TABLES}

Table 1 Distribution of severity scores and use of muscle relaxant in males and females

Table 1

Female

Male

$(\%)$

Total

Severity score

1

54 
(58.7)

38

(41.3)

92

2

398

(66.8)

198

(33.2)

596

3

328

(68.5)

151

(31.5)

479

4

53

(65.4)

28

(34.6)

81

5

8

(47.1)

9

(52.9)

17

Missing value

77

(67.5)

37

(32.5)

114 
Muscle relaxant

Yes

692

(67.6)

331

(32.4)

1023

No

185

(65.1)

99

(34.9)

284

Missing information

41

(56.9)

31

(43.1)

72

Total

918

(66.6)

461

(33.4)

1379

Table 2 Ratio of acute to baseline serum tryptase by age group in males and females

Age

All

$14-51$

+ As defined by acute total serum tryptase [?] $2+1.2$ baseline serum tryptase levels (baseline being lowest value between

Table 3 Ratio of acute to baseline serum tryptase for different severity scores 
Severity score+

1

2

3

4

5

Missing value

+ Modified Ring \& Messmer scale ++ Wilcoxon rank sum test with continuity correction + Modified Ring \& Messmer sc

Table 4 Temporal trends in total reports, NMBA use and IgE antibodies against Suxamethonium and Chlorhexidine

Women

Total

NMBA

IgE-SUX

IgE-HEX

Men

Total

NMBA

IgE-SUX

IgE-HEX

Sum of respective reporting variables per period. $\mathrm{RR}=$ relative risk of variable occurring compared with baseline period $\mathrm{P}$

\section{X: FIGURE LEGENDS}

Figure 1 Gender distribution in different age categories

Pearson's Chi-squared test: P-value $<.01$

Figure 2 Temporal trends in number of reports from 1997 to 2017, grouped by gender

Continuous line constructed using locally weighted scatterplot smoothing, grey area equals standard error. Sharp increase in incidence until 2001 is considered a consequence of increased reporting following the establishment of the Norwegian Network for Anaphylaxis under Anesthesia database. 

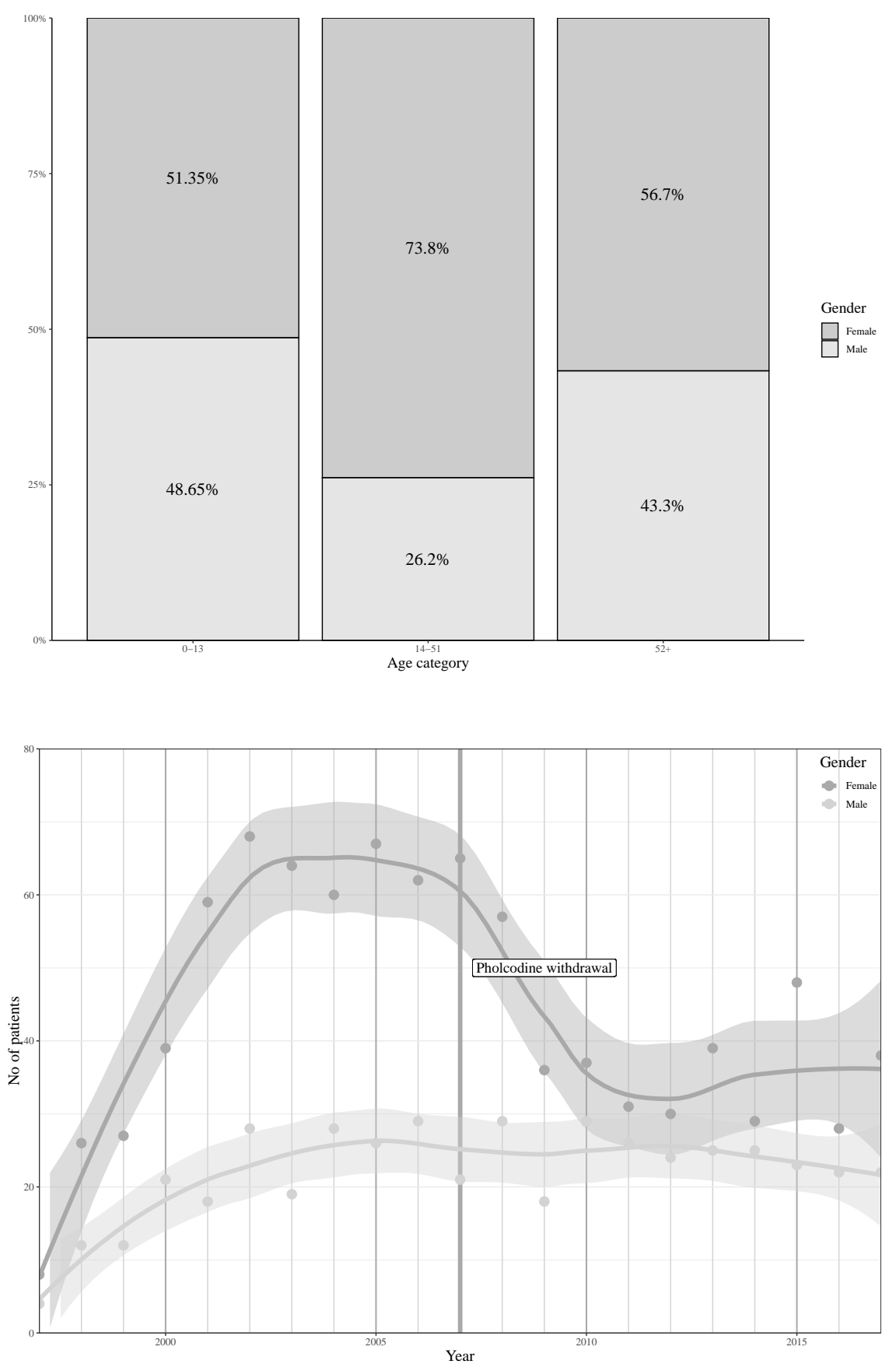